Revista de Psicología Vol. 33 (1), 2015 (ISSN 0254-9247)

\title{
Depresión en pacientes con esquizofrenia ingresados en servicios de agudos del Hospital Psiquiátrico de La Habana
}

\author{
Yasmani Llanes Basulto ${ }^{1}$, Yanquiel Barrios Hernández², Ignacio Oliva \\ Hernández ${ }^{3}$, Susel de la Caridad Pimentel Noda ${ }^{4}$ y Esvieta Calvo Guerra \\ Universidad de Ciencias Médicas de La Habana - Cuba
}

Se evalúa la presencia de depresión en la fase aguda de la esquizofrenia y se identifican las características clínicas y psicosociales que puedan estar asociadas a la depresión. Se estudiaron 73 pacientes ingresados en los servicios de agudos del Hospital Psiquiátrico de La Habana, identificándose que la depresión es un síntoma presente en una parte importante de los pacientes con esquizofrenia, el 35.6\% de la muestra presentó síntomas clínicamente significativos, y relacionados significativamente con la ausencia de vínculo laboral, la separación de seres queridos y un mayor número de ingresos. Es importante considerar la depresión y sus causas para desarrollar estrategias de tratamiento eficaces en este tipo de pacientes.

Palabras clave: depresión, esquizofrenia, servicios de agudos

1 Licenciado en Psicología por la Universidad de La Habana. Profesor instructor de la Facultad "Enrique Cabrera" de la Universidad de Ciencias Médicas de La Habana. Dirección postal: Calle 14 No. 108 entre 11 y 13, Plaza de la Revolución, La Habana, Cuba, CP 10400. Contacto: basulto@infomed.sld.cu

2 Licenciado en Psicología y máster en Ciencias Sociales por la Universidad de La Habana. Psicólogo clínico del Hospital Psiquiátrico de La Habana. Dirección postal: Calle 168 \#32503 Boyeros, La Habana. Cuba Contacto: yanquiel@infomed.sld.cu

3 Licenciado en Psicología por la Universidad de Ciencias Médicas de La Habana. Profesor instructor de la Facultad "Enrique Cabrera" de la Universidad de Ciencias Médicas de La Habana. Dirección postal: Cienfuegos 62 entre Corrales y Apodaca. CP 10200. Contacto: revistahph@infomed.sld.cu

4 Licenciada en Psicología por la Universidad de La Habana. Dirección postal: Edificio 46 A Apto. 1 calle 78 entre 5ta C y 5ta D, Villa Panamericana, Habana del Este, La Habana, CP.

5 Magíster en Medicinal Natural Tradicional y Bioenergética por la Universidad de Ciencias Médicas de La Habana. Profesora instructora de la Facultad "Enrique Cabrera" de la Universidad de Ciencias Médicas de La Habana. Dirección postal: CP 11400 Dc: 41 No. 5605 Apto. $10 / 56$ y 58 Playa. Contacto: evieteg@infomed.sld.cu 
Depression in patients with schizophrenia admitted to the acute services of the Psychiatric Hospital of Havana

The presence of depression in the acute phase of schizophrenia is evaluated, and the clini$\mathrm{cal}$ and psychosocial characteristics that can be associated with depression are identified. Participants included 73 patients that were admitted to the acute services of the Psychiatric Hospital of Havana, given that depression is a symptom in a significant amount of the patients with schizophrenia, $35.6 \%$ of the patients presented clinically significant symptoms, and these were related significantly with unemployment, the separation of loved ones and a greater number of re-hospitalizations. It is important to consider depression and its causes to develop effective treatment strategies.

Key words: depression, schizophrenia, acute services 
Los trastornos mentales son una problemática de gran magnitud para los sistemas de salud a nivel mundial. Si se compara a los trastornos mentales con otras enfermedades en relación con la invalidez que generan al individuo, los trastornos mentales poseen valores tan altos como los de las enfermedades cardiovasculares y las respiratorias e incluso sobrepasan a otras como diferentes tipos de cáncer (Bedirhan, 1999). Al mismo tiempo, se calcula que la depresión unipolar será para el 2020 la segunda causa de invalidez después de la enfermedad isquémica del corazón (Murray \& López, 1997). Asimismo, las proyecciones mundiales de la Organización Mundial de la Salud (OMS) identifican que la depresión unipolar será para el 2030 la principal causa de carga de enfermedad (OMS, 2004). Se considera también que los trastornos depresivos presentan altos índices de mortalidad y morbilidad, que deterioran seriamente la capacidad de funcionalidad social y laboral, en el último aspecto propiciando ausentismo que llevan al desempleo (Lépine \& Briley, 2011).

En varias condiciones la depresión es una comorbilidad común, este es el caso de la enfermedad cardiovascular (Sun, 2014), la artritis (Murphy, Sacks, Brady, Hootman \& Chapman, 2012), la enfermedad pulmonar obstrusiva crónica (Yong, Oak, Jin \& Hyun, 2010), en adultos mayores con deterioro cognitivo leve (Hidaka et al., 2012), en la diabetes (Aujla, Skinner, Khunti \& Davies, 2010; Poulsen \& Pachana, 2012), en la epilepsia (Fuller \& Brennenstuhl, 2009), en pacientes positivos al virus de inmunodeficiencia humana (Arseniou, Arvaniti \& Samakouri, 2014) y en pacientes con cáncer (Krebber et al., 2014). De manera general la depresión al estar presente en estas condiciones médicas proporciona un peor pronóstico, afecta seriamente la calidad de vida de los pacientes e impide un afrontamiento adecuado ante el diagnóstico y/o evolución de la enfermedad. 
En el caso de la comorbilidad de la depresión con otros trastornos mentales probablemente la mayor asociación sea con la ansiedad, de hecho se ha encontrado triple comorbilidad de ansiedad, depresión y trastorno por estrés postraumático, siendo este último un predictor de la ansiedad y la depresión. También se ha identificado la comorbilidad solo entre el trastorno por estrés postraumático y la depresión (Ginzburg, Ein-Dorb \& Solomon, 2010; Ikin, Creamer, Sim \& McKenzie , 2010; Rauch et al., 2010). Otros estudios han demostrado comorbilidad entre la depresión y el trastorno de ansiedad generalizada (Simon, 2009; Aragonès, Lluís \& Labad , 2009), con la fobia social (Ohayon \& Schatzberg , 2010), trastorno de pánico y trastorno de somatozación (Aragonès, Lluís Piñol \& Labad, 2009), con trastornos de la personalidad (Vilaplana, Mckenney, Riesco, Autonell \& Cervilla, 2010). Varios estudios también confirman la comorbildad de la depresión con las adicciones a sustancias (Torres, Posada Villa, Bareño \& Berbesí, 2010; Boden, Fergusson \& Horwood, 2010; Boden \& Fergusson, 2011). La evidencia muestra como la depresión parece acompañar a un grupo importante de afecciones mentales.

En el caso de la depresión en la esquizofrenia se ha identificado fuertes correlaciones, siendo este un tema polémico. Según la American Psychiatric Association (2013) entre las características clínicas de la esquizofrenia predominan los delirios, alucinaciones, leguaje desorganizado, conducta catatónica o groseramente desorganizada, así como la disminución de la expresión emocional, la pérdida de interés, el aplanamiento afectivo entre otros síntomas. Para el diagnóstico de la enfermedad, entre otros criterios, es necesario que estén presente dos o más de los síntomas antes mencionados, sin embargo existen otras alteraciones psíquicas que suelen estar presentes, aunque no sean determinantes para el diagnóstico según los manuales de clasificación; este es el caso de la depresión; una revisión reportada por Khouly, Mahmoud, Sadek y AlGafary (2012), refiere que tiene una prevalencia que fluctúa entre un 7 y $75 \%$.

Los trastornos depresivos y la esquizofrenia se conciben en las clasificaciones como padecimientos excluyentes (American Psychiatric 
Association, 2013), sin embargo los estudios demuestran que los síntomas depresivos aparecen en las diferentes fases de la esquizofrenia (Mauri, Paletta, Moliterno, Colasanti \& Altamura, 2010; D’Antonio \& Serper, 2012). En el periodo agudo, se han referido que existe mayor frecuencia, con una prevalencia entre un 22 y 80\% (DeLisi, 1990; Altamura et al., 1991; Siris et al., 2001, citados en Mauri, Paletta, Moliterno, Colasanti \& Altamura, 2010). Sin embargo, para otros autores, la depresión es una parte integral de la psicosis, que se mantiene durante todo el curso de la enfermedad (Häfner, an der Heiden \& Maurer, 2008). También se ha planteado la existencia de depresión en las fases iniciales de la enfermedad. Cotton y colaboradores (2012) comunican que el $26.2 \%$ de pacientes con trastorno del espectro esquizofrénico en su primer episodio psicótico presentan síntomas depresivos entre moderados y severos. Mientras que Sönmez y colaboradores (2014) refieren que en la fase inicial de la psicosis están presente hasta en un $41 \%$ de los pacientes y que de esta cantidad el 19\% se mantenía con síntomas depresivos 10 años después, las variables predictores de los síntomas depresivos en los pacientes que se mantenían después de 10 años fueron el pobre funcionamiento social durante la infancia y el consumo de alcohol.

Se plantea que los síntomas depresivos son frecuentes durante el primer episodio psicótico, que existe una tendencia a disminuir en los ańos siguientes pero que los pacientes con depresión en el primer episodio muestran peores resultados clínicos y funcionales que el resto (Sönmez et al., 2014). Por otra parte, la depresión en la esquizofrenia ha sido asociada con niveles bajos de autoestima, poca tolerancia a la frustración, propensión a la sobregeneralización, a autoculparse, evitar, fantaseo como un mecanismo de afrontamiento y menor uso de estrategias de solución de problemas y búsqueda de ayuda (Yan Xu et al., 2013). También existe relación entre los niveles de depresión e intensidad de las creencias estigmatizantes de los familiares cercanos, en pacientes con esquizofrenia con conciencia sobre su enfermedad (Krupchanka \& Katlia, 2013); otra investigación en este sentido encontró que pacientes con alto insight clínico tienen mayores puntuaciones en 
las escalas de depresión, lo cual además, se asocia a una mayor historia de intentos suicidas y sentimientos de desesperanza (Misdrahi, Denard, Swendsen, Jaussent \& Courtet, 2014).

Hay otras variables clínicas y psicosociales que suelen encontrarse como predictoras de los síntomas depresivos, estas son: ausencia de apoyo familiar, alta frecuencia de hospitalización, visitas infrecuentes de los familiares durante los ingresos, deterioro en la orientación así como la presencia de dolor (Chang \& Choi, 2012; Apiquian et al., 2001). Por último, la depresión, así como los síntomas negativos, han sido asociados con un menor bienestar emocional y social en pacientes en la fase crónica de la esquizofrenia (Straussa, Sandtb, Catalanoa \& Allenc, 2012). Aunque la esquizofrenia cuando se compara con otras enfermedades mentales tiene una relativa baja incidencia, y su prevalencia se calcula que oscila entre 4 y 7 por cada mil personas en el mundo, cuando aparece aun en sus formas más atenuadas se considera un trastorno mental altamente invalidante y muchas personas permanecen con síntomas persistentes a pesar de las mejores combinaciones de tratamientos existentes (Sukanta, Chant, Welham \& McGrath, 2005). Si a estas características de la enfermedad le adicionamos que con relativa frecuencia los síntomas depresivos acompañan su curso, entonces la invalidez y el sufrimiento para las personas que la padecen pueden ser mayores.

Dada la problemática reportada en la literatura respecto a la depresión en los pacientes con esquizofrenia y el hecho de no haberse encontrado estudios en pacientes cubanos, se decidió realizar la presente investigación de tipo descriptiva y transversal, para conocer la prevalencia y causas de esta alteración emocional en pacientes que ingresan en los servicios de agudos del Hospital Psiquiátrico de La Habana. Los objetivos planteados fueron evaluar la presencia de depresión en la fase aguda de la enfermedad e identificar las características clínicas y psicosociales que puedan estar asociadas a la depresión. Se considera que el presente estudio aporta información relevante que podría ser utilizada en el tratamiento psiquiátrico y psicoterapéutico de los pacientes con esquizofrenia y depresión. 


\section{Método}

\section{Participantes}

Se tomó una muestra del tipo no probabilística de pacientes que ingresaron en los servicios de agudos del Hospital Psiquiátrico de La Habana, durante los meses de enero y marzo de 2014. La muestra quedó constituida por 73 pacientes con un rango de edad entre 23 y 62 años, 35 del sexo femenino y 38 del sexo masculino.

Solo se incluyeron en el estudio los pacientes que cumplían con los siguientes criterios: (a) diagnóstico de esquizofrenia; (b) tiempo de hospitalización menor o igual a 7 días; y (c) otorgaron su consentimiento a participar en el estudio.

Se excluyeron del estudio a aquellos pacientes que: (a) presentaran problemas cognitivos severos que limitaran la comunicación y comprensión y (b) no cooperaron con el evaluador.

\section{Medidas e instrumentos de medición}

El diagnóstico psicopatológico de los participantes fue realizado con el Cuestionario para la Evaluación Clínica en Neuropsiquiatría versión 2.1 (SCAN-2.1). El sistema SCAN se compone de un conjunto de instrumentos y manuales destinados a analizar, medir, y clasificar la psicopatología y las principales alteraciones psiquiátricas de la edad adulta (Rijnders et al., 2000). En el presente estudio fue utilizado con el fin de realizar el diagnóstico de los pacientes, para lo cual se usaron las secciones 17 (Alucinaciones), 18 (Descripción subjetiva de trastornos del pensamiento y experiencias de reemplazo de la voluntad), 19 (Delirios), 20 (Información adicional necesaria para clasificar los trastornos de la Parte II), 22 (Ítems referentes a la actividad motora), 23 (Afectividad) y 24 (Alteraciones del lenguaje). El diagnóstico se obtuvo por medio de los algoritmos del DSM-IV.

La evaluación de la depresión se realizó con la Escala Calgary de Depresión en Esquizofrenia (Addington, Addington \& Maticka-Tyndale, 
1994; Addington, Addington, \& Schissel, 1990). La escala consta de nueve reactivos que se califican en un rango de severidad de 0 a 3 $(0=$ ausente y 3 = severo). La escala identifica la presencia de síntomas depresivos asociados a la esquizofrenia y los diferencia de los síntomas negativos y extrapiramidales.

Los datos psicosociales, sociodemográficos y clínicos de los participantes se recogieron a través de la Entrevista estructurada. La entrevista es una conversación que sostiene una persona con otra u otras con un propósito determinado. Por tanto, es un medio de comunicación entre ambas, en el que se manifiestan diferentes elementos: la palabra hablada, los ademanes, las expresiones, las inflexiones, etc. La entrevista puede clasificarse de diferentes formas, de acuerdo a su grado de estructuración pueden clasificarse en: estructuradas y no estructuradas o semiestructuradas. Las estructuradas son las que tienen un plan a seguir y aspectos a preguntar ya preparados (Alonso, Roja \& Cairo, 2003). En este estudio la entrevista se construyó con la finalidad de recoger los datos.

\section{Procedimiento}

$\mathrm{Al}$ incorporar a los pacientes para el estudio siempre se tuvo en cuenta que hubieran ingresado en un plazo no superior a 7 días. Se les explicó en qué consistía el estudio, que no se exponían a ningún riesgo para la salud, que se respetaría la privacidad y no se publicaría en modo alguno, información personal. Después se les pidió llenar un acta de consentimiento informado si deseaban participar y se procedía a aplicar las pruebas, comenzando por el SCAN para comprobar el diagnóstico de esquizofrenia, después se aplicaba la escala Calgary para depresión en esquizofrenia y por último la entrevista estructurada para obtener información sobre las siguientes variables: color de la piel, edad, sexo, vínculo laboral, cantidad de ańos escolares, estado civil, número de hijos, antecedentes patológicos familiares, años de evolución de la enfermedad, edad de inicio de la enfermedad, número de ingresos, seres queridos fallecidos, separación de seres queridos, y situaciones estresantes. 
El análisis estadístico se realizó mediante el programa SPSS versión 20.0. Se calcularon medidas de tendencia central y dispersión como la media y desviación estándar, así como frecuencias absolutas y relativas para describir la muestra según las variables clínicas y psicosociales incluidas en el estudio. Con el objetivo de identificar asociaciones entre la depresión y variables seleccionadas se empleó el Coeficiente de correlación por rangos de Spearman con un nivel de significancia de $p<.05$ y el coeficiente de contingencia, según se tratase de variables cualitativas ordinales o dicotómicas, respectivamente.

\section{Resultados}

\section{Depresión y características clínicas y psicosociales de la muestra}

La puntuación media en la Escala Calgary de Depresión en Esquizofrenia fue de 4.01 ( $D E=5.38)$. Las puntuaciones iguales o superiores a 6 son las que representan síntomas clínicamente significativos (Addington, Addington \& Schissel, 1990). El 35.6\% de los pacientes posee síntomas de depresión clínicamente significativos $(M=10.3$ puntos; $D E=4.13$ ). La mayor parte de los pacientes eran de raza blanca (50.7\%), le sucedió la raza negra (26\%) y mestiza (23.3\%). La mayoría se encontraban sin trabajar $(57.5 \%)$ y el resto realizaba alguna forma de actividad laboral (42.5\%). Respecto al estado civil se aprecia que la mayor parte estaba sin pareja (76.7\%), sea debido a que nunca se casaron o no tenían una relación en el momento de la evaluación (38.4\%), estaban divorciados $(17.8 \%)$, se separaron $(17.8 \%)$ o su pareja falleció $(6.8 \%)$; el resto $(23.3 \%)$ se encuentran acompańados $(11 \%)$ o casados (12.3\%).

El 45.2\% tenía antecedentes patológicos familiares de trastornos psiquiátrico; al 49.3\% se le había muerto algún ser querido, el 53.4\% vive separado de algún ser querido ya sea algún hijo, pareja anterior o hermanos, el $50.7 \%$ se encuentra atravesando una situación estresante la cual se asocia a conflictos familiares, problemas económico o a la crisis psiquiátrica actual y el $57.5 \%$ tienen hijos. 
Con relación a la escolaridad, los participantes presentaron una Media de 10.1 grados escolares $(D E=3.36$ años $)$ y la edad promedio de inicio de la enfermedad fue de 25.6 años $(M=25.6 ; D E=5.45$ años). Más aún, el promedio en años de evolución de la enfermedad hasta el momento de la investigación fue de 17.15 años $(M=17.5 ; D E=11.05$ años) y el valor medio de ingresos desde el inicio de la enfermedad hasta el presente fue de $8.18(D E=5.22)$.

\section{Relación entre la depresión y características clinicas y psicosociales de los pacientes}

Se observó asociación altamente significativa (ver Tabla 1) entre la depresión y el vínculo laboral (Coeficiente de contingencia .280; $p=.01$ ), siendo el porcentaje de pacientes con depresión y que no realizan actividad laboral (76.9\%). También se encontró asociación significativa entre la presencia de síntomas de depresión y la separación de seres queridos, donde el $69.2 \%$ de los sujetos deprimidos tuvieron que separarse de sus seres queridos (coeficiente de contingencia: .229; $p=.04)$. Se encontró una relación altamente significativa entre la severidad de las puntuaciones en ítems positivos en la Escala Calgary de Depresión en Esquizofrenia y el número de ingresos, o sea, a mayores valores en las puntuaciones en la escala mayor número de ingresos han tenido los pacientes $(R h o=.420 ; p=.000)$.

\section{Discusión}

Los resultados obtenidos en el presente estudio respecto al porcentaje de pacientes con esquizofrenia que presenta depresión concuerdan con los resultados de (DeLisi, 1990; Altamura et al., 1991; Siris et al., 2001, citados en Mauri et al., 2010), quienes señalan que la depresión está presente entre un 22 y $80 \%$ de los pacientes durante la fase aguda. Se considera que la depresión en la fase aguda es superior que en otros momentos del curso de la enfermedad. Esta muestra presentó un porcentaje de depresión superior al reportado por otra investigación con 
pacientes esquizofrénicos en la fase estable, donde la prevalencia fue de 31\% (Majadas, Olivares, Galan \& Diez, 2012).

\section{Tabla 1}

Relación entre la depresión y las variables estudiadas

\begin{tabular}{|c|c|c|c|}
\hline Variable & Prueba estadística & $\begin{array}{l}\text { Valor de la } \\
\text { Prueba }\end{array}$ & $p$ \\
\hline Color de la piel & Coeficiente de contingencia & .084 & .43 \\
\hline Edad & $\begin{array}{l}\text { Coeficiente de correlación por } \\
\text { rangos de Spearman }\end{array}$ & .229 & .05 \\
\hline Sexo & Coeficiente de contingencia & .084 & .43 \\
\hline Vínculo laboral & Coeficiente de contingencia & .280 & $.01^{*}$ \\
\hline Cantidad de ańos escolares & $\begin{array}{l}\text { Coeficiente de correlación por } \\
\text { rangos de Spearman }\end{array}$ & -.209 & .07 \\
\hline Estado civil & Coeficiente de contingencia & .193 & .09 \\
\hline $\begin{array}{l}\text { Antecedentes patológicos } \\
\text { familiares de trastorno } \\
\text { mental }\end{array}$ & Coeficiente de contingencia & .087 & .75 \\
\hline $\begin{array}{l}\text { Años de evolución de la } \\
\text { enfermedad }\end{array}$ & $\begin{array}{l}\text { Coeficiente de correlación por } \\
\text { rangos de Spearman }\end{array}$ & .199 & .09 \\
\hline Número de ingresos & $\begin{array}{l}\text { Coeficiente de correlación por } \\
\text { rangos de Spearman }\end{array}$ & .420 & $.00^{*}$ \\
\hline Seres queridos fallecidos & Coeficiente de contingencia & .179 & .12 \\
\hline Situaciones estresantes & Coeficiente de contingencia & .159 & .16 \\
\hline $\begin{array}{l}\text { Separación de seres } \\
\text { queridos }\end{array}$ & Coeficiente de contingencia & .229 & $.04^{*}$ \\
\hline
\end{tabular}

Nota: ${ }^{*} p<.05$

Por otra parte, la depresión en pacientes con esquizofrenia es mucho mayor a la que se encuentra en otras poblaciones, por ejemplo, la prevalencia del trastorno depresivo unipolar en la población general 
es de 4.4\% (World Health Organization, 2002), en el presente estudio los síntomas de depresión clínicamente significativos superan a esta estadística en más de 8 veces. Al comparar este grupo de pacientes con la depresión que se encuentra en diferentes tipos de condiciones físicas los valores pueden ser mayores. Los porcentajes de la prevalencia de la depresión fueron en la enfermedad cardiovascular (21.7\%); en la enfermedad cerebrovascular (25.5\%) (Sun, 2014); en la enfermedad pulmonar obstrusiva crónica (15.4\%) (Yong, Oak, Jin \& Hyun, 2010); en la epilepsia (13\%) (Fuller \& Brennenstuhl, 2009); en pacientes con cáncer de próstata (8.54\%) (Jayadevappa, Malkowicz, Chhatre, Johnson \& Gallo, 2012).

El grupo de pacientes analizados en este estudio también presentó un mayor porcentaje con síntomas de depresión clínicamente significativos al compararlo con lo que refieren otros estudios con distintas alteraciones mentales. En los trastornos de personalidad los valores de la depresión fueron para los pacientes con trastornos del cluster C (17\%), seguido del cluster A (10\%) y el cluster B (9.8\%) (Vilaplana, Mckenney, Riesco, Autonell \& Cervilla, 2010); en el trastorno de pánico (33.8\%), en el trastorno de somatización (6.6\%) (Aragonès et al., 2009); en la fobia social (19.5\%) (Ohayon \& Schatzberg, 2010).

La evidencia al comparar con los estudios antes mencionados tanto con condiciones físicas como con alteraciones mentales demuestran que la depresión y la esquizofrenia tienen niveles de asociación importante, que pueden llegar a ser superiores a la depresión que acompaña a alguna otra condición de salud.

La comorbilidad entre la depresión y otras condiciones de salud en mayor medida hace referencia a un síntoma que acompaña. En muchos casos puede ser una reacción del organismo a un evento negativo como lo es la adquisición de una enfermedad más que ser una cualidad intrínseca de la enfermedad. La presencia de depresión ante estímulos estresores o negativos hace referencia a las condiciones de la personalidad para afrontar el acontecimiento. 
Esto puede ser explicado desde varios conceptos que hoy en día constituyen claves de la psicología de la salud para trazarse modelos explicativos del proceso salud-enfermedad, en este sentido se hace referencia a los conceptos de atribución causal, locus de control, o resiliencia. El concepto de atribución causal hace referencia al proceso mediante el cual las personas establecen los componentes causales de determinado hecho que tiene lugar en su vida o entorno. Una persona que atribuye sus éxitos a causas externas no controlables y además se culpa de sus fracasos, atribuyéndolos a causas internas sobre las que tampoco ejerce un control es propensa a desarrollar depresiones (Roca, 2013).

El locus de control externo que sería el más relacionado en estos casos tiene que ver con un peor afrontamiento a la enfermedad en tanto se le atribuyen causas externalizantes, la persona no se implica responsablemente para hacerle frente, una persona con locus de control externo está centrada en las limitaciones de la enfermedad, en el problema; mientras que una con un locus de control interno estaría centrada en las soluciones y en la posibilidades que aún le quedan. Luego, estas características o estilos para afrontar la enfermedad tienen relación estrecha con la historia de vida de la persona, con sus aprendizajes y hacen referencia al robustecimiento de la personalidad o sea a la resiliencia que tiene que ver con la capacidad de la persona para superar las situaciones no deseadas de la vida, mantener y continuar viviendo con calidad aun con la presencia de una enfermedad crónica (Roca, 2013).

En el caso de la esquizofrenia la depresión podría ser en algunos casos una cualidad propia de la enfermedad que se deba a mecanismos neurobioquímicos o genéticos implicados en esta alteración, sin embargo son innegable las disfunciones psicosociales en la mayoría de estos pacientes, como pérdida del empleo, divorcios, falta de apoyo social, rechazo familiar, alta estima autopercibida y también un debilitamiento de los mecanismos de la personalidad para afrontar los eventos negativos; todos estos factores claramente pueden llevar al paciente a frecuentes depresiones. 
Varios estudios confirman un amplio grupo de variables psicosociales asociadas a la depresión en la esquizofrenia, entre estas: niveles bajos de autoestima, poca tolerancia a la frustración, propensión a la sobregeneralización, a autoculparse, fantaseo como un mecanismo de afrontamiento, menor uso de estrategias de solución de problemas y búsqueda de ayuda (Yan Xu et al., 2013), intensidad de las creencias estigmatizantes de los familiares cercanos (Krupchanka \& KatliaR, 2013), con alto insight clínico, mayor historia de intentos suicidas y sentimientos de desesperanza (Misdrahi et al., 2014), alta frecuencia de hospitalización (An der Heiden, Könnecke, Maurer \& Ropeter, 2005), ausencia de apoyo familiar, visitas infrecuentes de los familiares durante los ingresos, deterioro en la orientación (Chang \& Choi, 2012; Apiquian et al., 2001), incremento del riesgo de suicidio (Prasad, 1986) y menor bienestar emocional y social (Straussa, Sandtb, Catalanoa \& Allenc, 2012). En este estudio se encontró relación con ausencia de vínculo laboral, la separación de seres queridos y un mayor número de ingresos.

En el caso de la relación con el vínculo laboral, podría ser explicado desde varias perspectivas. Los pacientes que tienen depresión tienen un cuadro clínico más severo y limitante y una menor motivación por emplearse. También la depresión puede ser lo opuesto a lo antes referido, o sea, el efecto y no la causa en relación con el desempleo, el hecho de no obtener un empleo los limita de adquirir los recursos económicos para satisfacer las necesidades materiales personales y familiares y por ende reaccionan ante esta situación social presentando este síntoma. Esta relación entre la depresión y el desempleo en los casos más extremos puede llevar al paciente al suicidio, una investigación en este sentido encontró que en el $80 \%$ de pacientes esquizofrénicos que se suicidaron existía un vínculo con la depresión y el desempleo (Roy, 1982).

Respecto a la separación de seres queridos en esta muestra se aprecia que las personas a que más hacen referencia son los hijos y parejas como seres de los que se ha tenido que separar. Para estos pacientes estructurar su vida en ausencia de figuras con las cuales muestran un vínculo afectivo importante puede ser un resorte de este estado afectivo, 
así como el hecho de que vivan sin la presencia de estas personas hace que posean menos apoyo social. Se ha identificado que la depresión puede asociarse a la falta de pareja, lo cual puede incluso ir acompañado de ideación suicida (Apiquian, et al., 2001; Kasckow et al., 2010).

En este estudio, los pacientes que han tenido más recaídas o ingresos poseen mayor cantidad e intensidad de los síntomas depresivos. Un mayor número de ingresos o rehospitalizaciones tiene que ver con una cantidad mayor de recaídas, lo que puede ser por una severidad mayor de la enfermedad o una mayor cantidad de síntomas psicóticos (Li Ning, Rondón, Luzmina \& Ballón, 2003); y la depresión integrarse como un síntoma más del espectro de síntomas propios de la esquizofrenia; aunque también los síntomas depresivos pueden asociarse a los ingresos porque los pacientes con más ingresos puede que tengan menos redes de apoyo, lo que hace que se descompensen más y que la depresión sea más bien una reacción ante las condiciones de existencia del paciente, como el propio hecho de no tener empleo, o estar separado de sus seres queridos. Los autores del trabajo sostienen el criterio que son las variables psicosociales las que más inciden en estos pacientes.

En esta muestra, la depresión no se encontró relacionada con otras variables como el color de la piel, el sexo, el estado civil, los antecedentes patológicos familiares de trastornos mentales, los seres queridos fallecidos, las situaciones estresantes, años de evolución desde el inicio de la enfermedad hasta el momento de la investigación, ni con la cantidad de años escolares.

Entre las principales limitaciones del estudio pueden referirse no considerar otras variables que podrían estar asociadas a la depresión, ya que, en su aparición pueden incidir factores biológicos, genéticos y psicológicos. Aquí se ha demostrado relación de la depresión con condiciones fundamentalmente ambientales que inciden sobre el paciente, sin embargo, vale mencionar que debe prestarse atención en estudios posteriores, al valor de las condiciones internas subjetivas como las experiencias anteriores de los pacientes, estrategias de afrontamiento, características de la personalidad, etc. que marcan la diferencia con los que en situaciones estresoras o desfavorables similares no se deprimen. 
En conclusión puede afirmarse, como señalan otros autores, que la depresión es un síntoma identificable en una parte importante de los pacientes con esquizofrenia, en este caso los ingresados en servicios de agudos, asociándose fundamentalmente a variables psicosociales como pueden ser la ausencia de vínculo laboral, la separación de seres queridos y la cantidad de ingresos.

Los resultados pueden tener varias implicaciones clínicas, no solo se limitan al conocimiento de las particularidades de la psicopatología de estos pacientes, sino también que pueden ser útiles para diseñar intervenciones que sean más efectivas. Se considera que el abordaje terapéutico del paciente debe ser multidisciplinario y contar además, con la colaboración de los trabajadores sociales para lograr vincular a los pacientes a empleos según las capacidades que conservan, así como incluir también, intervenciones psicoeducativas dirigidas a la familia.

\section{Referencias}

Addington, D., Addington, J. \& Maticka-Tyndale, E. (1994). Specificity of the Calgary Depression Scale for schizophrenics. Schizophrenia research, 11(3), 239-244.

Addington, D., Addington, J. \& Schissel, B. (1990). A depression rating scale for schizophrenics. Schizophrenia research, 3(4), 247-251.

Alonso, A., Roja, R. \& Cairo, E. (2003). Selección de lecturas de psicodiagnóstico. La Habana: Editorial Félix Varela.

American Psychiatric Association. (2013). Diagnostic and Statistical Manual of Mental Disorders (Fifth ed.). Washington DC-London: American Psychiatric Publishing.

An der Heiden, W., Könnecke, R., Maurer, K. \& Ropeter, D. (2005). Depression in the long-term course of schizophrenia. European archives of psychiatry and clinical neuroscience, 255(3), 174-184. Apiquian, R., Fresán, A., Ulloa, R., García, M., Lóyzaga, C., Nicolini, H. \& Ortega, H. (2001). Estudio comparativo de pacientes esquizofrénicos con y sin depresión. Salud Mental, 24(5), 25-29. 
Aragonès, E., Lluís Piñol, J. \& Labad, A. (2009). Comorbilidad de la depresión mayor con otros trastornos mentales comunes en pacientes de atención primaria. Atención Primaria, 41(10), 545551.

Arseniou, S., Arvaniti, A. \& Samakouri, M. (2014). HIV infection and depression. Psychiatry and Clinical Neurosciences, 68(2), 96-109. Aujla, N., Skinner, T., Khunti, K. \& M. J. Davies, M. (2010). The prevalence of depressive symptoms in a white European and South Asian population with impaired glucose regulation and screen-detected Type 2 diabetes mellitus: a comparison of two screening tools. Diabetic Medicine, 27(8), 896-905.

Bedirhan, U. (1999). The Global Burden of Mental Disorders. American Journal of Public Health, 89(9), 1315-1318.

Boden, J. M., Fergusson, D. M. \& Horwood, J. (2010). Cigarette smoking and depression: tests of causal linkages using a longitudinal birth cohort. The British Journal of Psychiatry, 196(6), 440-446.

Boden, J. \& Fergusson, D. (2011). Alcohol and depression. Addiction, 106(5), 906-914.

Chang, S. \& Choi, T. (2012). Prevalence and predictors of depression among the schizophrenia patients in chronic institution. European Psychiatry, 27(1), 1-1.

Cotton, S., Lambert, M., Schimmelmann, B., Mackinnon, A., Gleeson, J., Berk, M., . . \& \& Conus, P. (2012). Depressive symptoms in first episode schizophrenia spectrum disorder. Schizophrenia Research, 134(1), 20-26.

D'Antonio, E. \& Serper, M. (2012). Depression and cognitive deficits in geriatric schizophrenia. Schizophrenia Research, 134(1), 65-69.

Fuller, E. \& Brennenstuhl, S. (2009). The association between depression and epilepsy in a nationally representative sample. Epilepsia, 50(5), 1051-1058.

Ginzburg, K., Ein-Dorb, T. \& Solomon, Z. (2010). Comorbidity of posttraumatic stress disorder, anxiety and depression: A 20-year longitudinal study of war veterans. Journal of Affective Disorders, 123(1-3), 249-257. 
Häfner, H., an der Heiden, W. \& Maurer, K. (2008). Evidence for separate diseases?. European archives of psychiatry and clinical neuroscience, 258(2), 85-96.

Hidaka, S., Ikejima, C., Kodama, C., Nose, M., Yamashita, F., Sasaki, M., . . . \& Asada, T. (2012). Prevalence of depression and depressive symptoms among older Japanese people: comorbidity of mild cognitive impairment and depression. Int $J$ Geriatr Psychiatry, 27(3), 271-279.

Ikin , J., Creamer , M., Sim, M. \& McKenzie, D. (2010). Comorbidity of PTSD and depression in Korean War veterans: Prevalence, predictors, and impairment. Journal of Affective Disorders, 125(1-3), 279-286.

Jayadevappa, R., Malkowicz, S. B., Chhatre, S., Johnson, J. C. \& Gallo, J. J. (2012). The burden of depression in prostate cancer. Psycho-Oncology, 21(12), 1338-1345.

Kasckow, J., Fellows, I., Lawrence, C. E., Golshan, S., Solorzano, E. \& Zisook, S. (2010). The relationship of marital status and clinical characteristics in middle-aged and older patients with schizophrenia and depressive symptoms. Annals of clinical psychiatry, 22(3), 172-179.

Khouly, G., Mahmoud, A., Sadek, H. \& AlGafary, M. (2012). A study compared Hamilton and Calgary depressive scales in assessing depression in schizophrenia. European Psychiatry, 27(1), 1.

Krebber, A., Buffart, L., Kleijn, G., Riepma, I., Bree, R., Leemans, C., .. . \& Verdonck-de Leeuw, I. (2014). Prevalence of depression in cancer patients: a meta-analysis of diagnostic interviews and self-report instruments. Psycho-Oncology, 23(2), 121-130.

Krupchanka, D. \& Katlia, R. M. (2013). Insight mediates link between stigma and depression in schizophrenia. European Psychiatry, 28, $1-1$.

Lépine, J. \& Briley, M. (2011). The increasing burden of depression. Neuropsychiatric Disease and Treatment, 7(1), 3-7. 
Li Ning, J., Rondón, M., Luzmina, R. \& Ballón, R. (2003). Causas de rehospitalización de pacientes esquizofrénicos crónicos. Revista de Neuro-psiquiatria, 66(1), 13-26.

Majadas, S., Olivares, J., Galan, J. \& Diez, T. (2012). Prevalence of depression and its relationship with other clinical characteristics in a sample of patients with stable schizophrenia. Comprehensive Psychiatry, 53(2), 145-151.

Mauri, M., Paletta, S., Moliterno, D., Colasanti, A. \& Altamura, C. (2010). Depressive dimension, clinical outcome, and duration of hospitalization in acute schizophrenia. Asian Journal of Psychiatry, 3(3), 121-125.

Misdrahi, D., Denard, S., Swendsen, J., Jaussent, I. \& Courtet, P. (2014). Depression in schizophrenia: The influence of the different dimensions of insight. Psychiatry Research, 216(1), 12-16.

Murphy, L., Sacks, J., Brady, T., Hootman, J. \& Chapman, D. (2012). Anxiety and Depression Among US Adults With Arthritis: Prevalence and Correlates. Arthritis Care \& Research, 64(7), 968-976.

Murray, C. \& Lopez, A. (1997). Alternative projections of mortality and disability by cause 1990-2020: Global Burden of Disease Study. The Lancet, 349(9064), 1498-1504.

Ohayon, M. M. \& Schatzberg, A. F. (2010). Social phobia and depression: Prevalence and comorbidity. Journal of Psychosomatic Research, 68(3), 235-243.

OMS (2004). The global burden of disease: 2004 update. Recuperado de http://www.who.int/entity/healthinfo/global_burden_disease/

Poulsen, K. \& Pachana, N. (2012). Depression and Anxiety in Older and Middle-aged Adults With Diabetes. Australian Psychologist, 47(2), 90-97.

Prasad, A. (1986). Attempted suicide in hospitalized schizophrenics. Acta Psychiatr Scand, 74(1), 41-42.

Rauch, S., Favorite, T., Giardino, N., Porcari, P., Defever, E. \& Liberzon, I. (2010). Relationship between anxiety, depression, and health satisfaction among veterans with PTSD. Journal of Affective Disorders, 121(1-2), 165-168. 
Rijnders, C. T., Van den Berg, J. F., Hodiamont, P. P., Nienhuis, F. J., Furer, J. W. \& Mulder, J. (2000). Psychometric properties of the schedules for clinical assessment in neuropsychiatry (SCAN-2.1). Social psychiatry and psychiatric epidemiology, 35(8), 348-352.

Roca, M. A. (2013). Psicología clínica. Una mirada desde la salud bumana. La Habana: Editorial Félix Varela.

Roy, A. (1982). Suicide in chronic schizophrenia. The British Journal of Psychiatry, 141(2), 171-177.

Simon, N. M. (2009). Generalized anxiety disorder and psychiatric comorbidities such as depression, bipolar disorder, and substance abuse. Journal of Clinical Psychiatry, 70(2), 10-14.

Sönmez, N., Røssberg, J. I., Evensen, J., Eidsmo, H., Haahr, U., ten Velden, W., ... \& Friis, S. (2014). Depressive symptoms in firstepisode psychosis: a 10-year follow-up study. Early Intervention in Psychiatry. doi:10.1111/eip.12163.

Straussa, G., Sandtb, A., Catalanoa, L. \& Allenc, D. (2012). Negative symptoms and depression predict lower psychological well-being in individuals with schizophrenia. Comprehensive Psychiatry, 53(8), 1137-1144.

Sukanta, S., Chant, D., Welham, J. \& McGrath, J. (2005). A Systematic Review of the Prevalence of Schizophrenia. PLoS Medicine, 2(5), e141.

Sun, E. S. (2014). Cardiovascular disease risk factors associated with depression among Korean adults with coronary artery disease and cerebrovascular disease. Asia-Pacific Psychiatry. doi:10.1111/ appy. 12139

Torres, Y., Posada Villa, J., Bareño Silva, J. \& Berbesí Fernández, D. Y. (2010). Trastornos por abuso y dependencia de sustancias en población colombiana: su prevalencia y comorbilidad con otros trastornos mentales seleccionados. Revista Colombiana de Psiquiatría, 39(Supl.), 14-35.

Vilaplana, M., Mckenney, K., Riesco, M. D., Autonell, J. \& Cervilla, J. A. (2010). Comorbilidad depresiva en los trastornos de la personalidad. Revista de Psiquiatría y Salud Mental, 3(1), 4-12. 
World Health Organization. (2002). The World Health. Geneva: WHO. Yan Xu, Z., Zu, S., TaoXiang, Y., Wang, N., HuaGuo, Z., Kilbourne, A., . . \& JiangLi, Z. (2013). Associations of self-esteem, dysfunctional beliefs and coping style with depression in patients with schizophrenia: A preliminary survey. PsychiatryResearch, 209(3), 340-345.

Yong, S., Oak, W., Jin, B. \& Hyun, M. (2010). Relationship between depression and health-related quality of life in older Korean patients with chronic obstructive pulmonary disease. Journal of Clinical Nursing, 19(9-10), 1307-1314.

Recibido: 17 de julio, 2014 Aceptado: 1 de septiembre, 2014 